\title{
Detailed multigrade evaluation of fetal disorders with the quantified actocardiogram
}

\author{
Kazuo Maeda', ${ }^{1, *}$, Tomio Iwabe ${ }^{1}$, Souichi \\ Yoshida', Takashi Ito', Yukihisa Minagawa', \\ Seiichi Morokuma ${ }^{2}$, Ritsuko K. Pooh ${ }^{3}$ and \\ Taisuke Fuchiwaki ${ }^{4}$ \\ ${ }^{1}$ Department of Obstetrics and Gynecology, Tottori \\ University School of Medicine, Yonago, Japan \\ 2 Department of Obstetrics and Gynecology, Kyushu \\ University, Graduate School of Medical Science, \\ Fukuoka, Japan \\ ${ }^{3}$ CRIFM Clinical Research Institute of Fetal Medicine, \\ PMC, Osaka, Japan \\ ${ }^{4}$ Urayasu-Ichikawa Municipal Hospital, Urayasu, Japan
}

\begin{abstract}
Aims: To evaluate fetal disorders using detailed quantitative values from the actocardiogram (ACG) involving simultaneous tracing of ultrasonic Doppler fetal movement bursts and fetal heart rate (FHR).

Methods: Duration of FHR accelerations and fetal movement bursts were measured manually in 20 common fetal disorders. The severity of the fetal disorder was estimated using the FHR acceleration duration to movement burst ratio ( $A / B$ ratio) and $10-0$ clinical severity ranks derived from the $A / B$ ratio. The correlation of the $A / B$ ratio and 1 and 5 min Apgar scores, as well as numerically expressed long-term outcomes were studied.

Results: A/B ratios were significantly correlated with the 1 and 5 min Apgar scores and the numerically evaluated long-term outcomes. Controversial cases of FHR pattern were more easily understood using the $A / B$ ratio. The 10-0 severity derived from the $A / B$ ratio was useful in clinical fetal studies.

Conclusion: Common fetal disorders were evaluated quantitatively and in more detail using the $A / B$ ratio from the actocardiogram than when using common binary good or bad evaluation. The A/B ratio was useful in outcome estimation, where the prognostic capability of the $\mathrm{A} / \mathrm{B}$ ratio was confirmed by significant correlation with 1 and 5 min Apgar scores and long-term outcomes of fetal disorders.
\end{abstract}

\footnotetext{
${ }^{*}$ Corresponding author:

Kazuo Maeda, MD

Department of Obstetrics and Gynecology (Prof.emeritus)

Tottori University

Nadamachi 3-125

Yonago, Tottoriken, 683-0835

Japan

E-mail: maedak@mocha.ocn.ne.jp
}

Keywords: Actocardiogram; fetal disorder; fetal movement; FHR acceleration; quantified evaluation; ultrasound.

\section{Introduction}

The severity of fetal disorders is commonly classified into two gross grades, normal and ominous. For example, reactive acceleration might be normal and non-reactive NST might be ominous [17]. However, multiple grades between normal acceleration and its disappearance must exist. Detailed evaluation of fetal severity was accomplished in fetal CNS lesions by quantitative studies on the ratio of FHR acceleration duration to movement burst duration ( $A / B$ ratio) by quantified ACG, where a regression equation of fetal $A / B$ ratio and severity was proposed [11]. In this paper, we evaluate common fetal disorders using the $A / B$ ratio and correlate the $A / B$ ratio with Apgar scores and long-term outcome.

\section{Methods}

The ACG was recorded with various models of actocardiograph produced in uniform quality to record of fetal movement. The models were the hand-made prototype by KM [7], MT-320, MT325, MT-332, MT-333U, MT-430, MT-516, MT-517, MT-522 and MT-540 (TOITU, Tokyo). Fetal movement and fetal heart beat were detected using a single ultrasound transducer positioned on the maternal abdomen to detect a clear fetal heart beat. FHR was traced in the upper part of the CTG chart, and fetal movement bursts were recorded in the lower part (Figure 1). Durations of fetal movement bursts composed of clustered spikes and those of FHR accelerations were measured manually and analyzed [8-11]. Fetal behavioral states, fetal hiccups, physiological sinusoidal FHR accompanied by periodic fetal movements in fetal breathing and mouthing movements were determined by the ACG [8, 10].

Movement spikes of the ACG in late pregnancy were recorded simultaneously with event markers of fetal trunk movement observed by real time B-mode, where sonographically detected movement marks were concordant with movement spikes on the ACG [11]. Accuracy of movement bursts recorded with spikes and dots were compared. Since the movement duration was shorter, interval was longer and movement burst count was less in dot records [11], and a report criticized the inaccuracy of ACG in a dot-recording machine [2], spike records were used in quantitative studies on ACGs. The accuracy of ACGs to record $95-100 \%$ of fetal trunk movements was established $[1,3,15,18]$.

The duration of every movement burst and FHR acceleration were measured manually using the chart time scale and their 


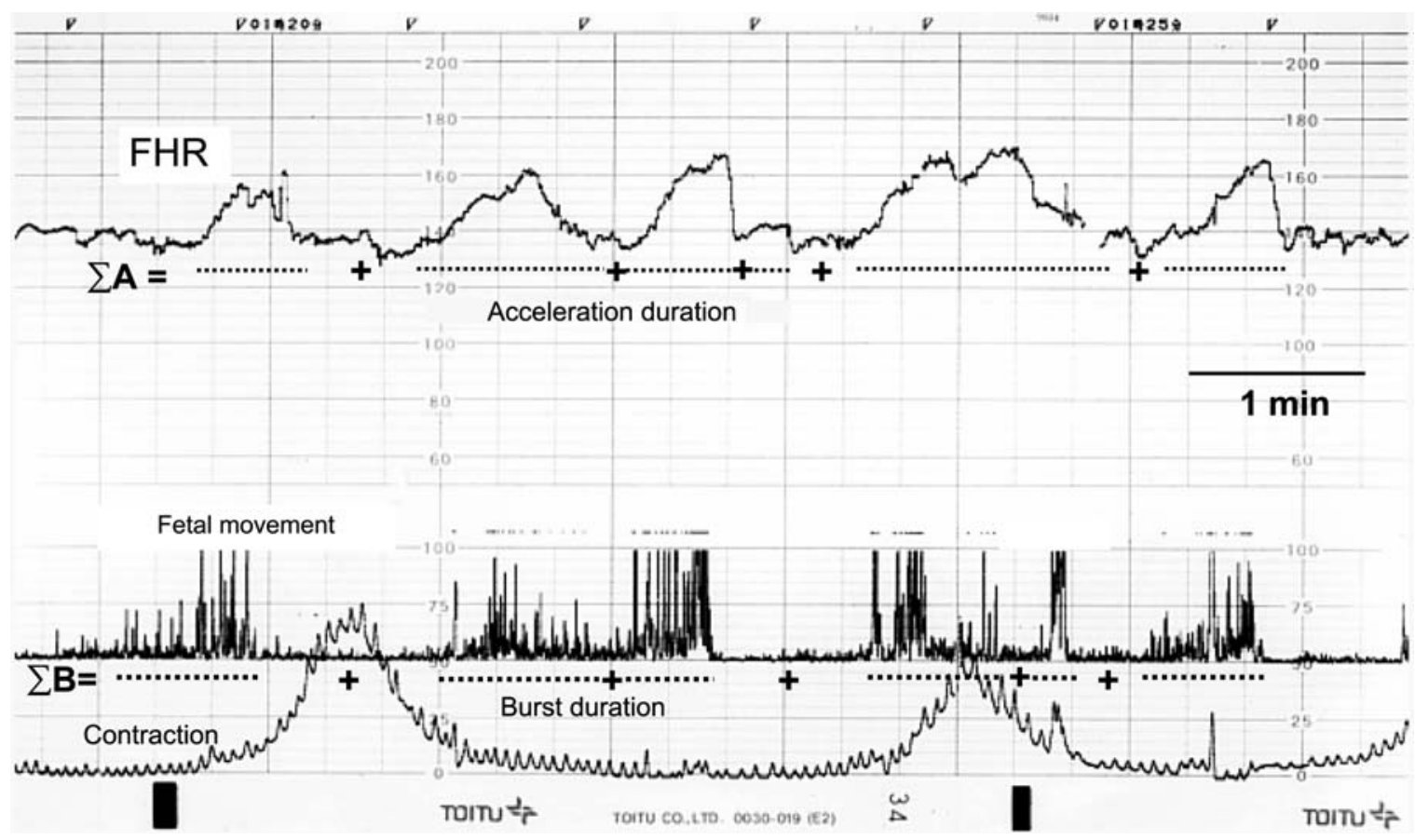

Figure 1 It is an example of actocardiogram $(A C G)$ in the active fetal state. The $A / B$ ratio $=\Sigma A / \Sigma B$, i.e., the sum of $F H R$ acceleration duration $(\Sigma A)$ is divided by the sum of fetal movement burst duration $(\Sigma B)$ in the same periods in the ACG.

relationships were studied $[10,17]$. The tested parameters were the mean movement burst duration (s), occupancy (\%), frequency (cpm), FHR acceleration duration and number ratio to movement burst (A/B duration and number ratio). Two $A / B$ ratios were significantly smaller in fetal central nervous system lesions than in normal pregnancies [11]. The A/B duration ratio was used and called simply $A / B$ ratio in this report.

Nineteen fetal disorders and one normal pregnancy at 26-41 weeks' gestation were studied, including non-reassuring fetal status (NRFS), aneuploidy, myotonic dystrophy, fetal

Table 1 Fetal disorders, the A/B ratio of ACG, 1 and 5 min Apgar scores, and numerically evaluated long-term outcome (Table 2) in the cases studied in this report.

\begin{tabular}{|c|c|c|c|c|c|}
\hline \multirow[t]{2}{*}{ Fetal disorders } & \multirow{2}{*}{$\begin{array}{l}\text { Weeks of } \\
\text { pregnancy }\end{array}$} & \multirow{2}{*}{$\begin{array}{l}\mathrm{A} / \mathrm{B} \\
\text { ratio }\end{array}$} & \multicolumn{2}{|c|}{ Apgar score } & \multirow{2}{*}{$\begin{array}{l}\text { Long-term } \\
\text { outcome }\end{array}$} \\
\hline & & & $1 \mathrm{~min}$ & $5 \mathrm{~min}$ & \\
\hline NRFS (LOV) & 35 & 0 & 0 & 0 & 0 \\
\hline 18-trisomy & 29 & 0.15 & 0 & 0 & 0 \\
\hline Fetal hydrops & 26 & 0.42 & 0 & 0 & 0 \\
\hline Osteodysplasia & 29 & 0.50 & 1 & 4 & 1 \\
\hline Excencephaly, multiple anomalies & 37 & 0.54 & 1 & 3 & 1 \\
\hline Intestinal obstruction & 36 & 0.96 & 2 & 4 & 6 \\
\hline Polydactyly & 37 & 1.01 & 8 & 9 & 9 \\
\hline Cardiac sick sinus syndrome & 39 & 1.34 & 7 & 9 & 9 \\
\hline Endocardiac cushion defect & 37 & 1.20 & 9 & 9 & 8 \\
\hline Megacystis & 38 & 1.22 & 8 & 9 & 8 \\
\hline Myotonic dystrophy & 35 & 1.28 & 9 & 9 & 8 \\
\hline Hydronephrosis & 39 & 1.31 & 9 & 9 & 9 \\
\hline Normal pregnancy & 37 & 1.43 & 8 & 9 & 10 \\
\hline Hydrocephaly & 33 & 1.16 & 8 & 9 & 7 \\
\hline Hydrocephaly & 30 & 0.73 & 3 & 4 & 4 \\
\hline Spina bifida, ventriculomegaly & 41 & 0.88 & 4 & 8 & 6 \\
\hline Spina bifida, partial CCD & 34 & 1.11 & 8 & 8 & 8 \\
\hline Aortic arch interruption, type A & 40 & 1.34 & 8 & 9 & 8 \\
\hline IUGR, NRFS (LD) & 35 & 0.34 & 2 & 9 & 8 \\
\hline NRFS (LD), placental abruption & 38 & 1.32 & 9 & 9 & 9 \\
\hline
\end{tabular}

$\mathrm{NRFS}=$ non-reassuring fetal status, $\mathrm{LOV}=$ loss of variability, $C C D=$ corpus callosum defect, IUGR $=$ intrauterine growth restriction, $\mathrm{LD}=$ late decelerations. 
Table 2 Numerical evaluation of the long-term fetal outcome.

\begin{tabular}{lc}
\hline Long-term outcome & $\begin{array}{c}\text { Numerical } \\
\text { evaluation }\end{array}$ \\
\hline Intrauterine death & 0 \\
Death on day 1 & 1 \\
Death within 1 week & 2 \\
Death within 1 year & 3 \\
Death under 5 years & 4 \\
Spastic quadriplegia & 5 \\
Growth retardation & 6 \\
Mental retardation & 7 \\
Well and change hospital & 8 \\
Well and mild sickness & 9 \\
Healthy & 10 \\
\hline
\end{tabular}

hydrops, excencephaly, hydrocephaly, spina bifida, congenital heart diseases, polydactyly, megacystis, bradycardia due to cardiac sick sinus syndrome and placental abruption. Their ACGs were registered as the non-stress test during pregnancy (Table 1). The ACGs of 20 cases were collected by the authors, all of the durations of FHR accelerations and fetal movement burst were manually measured and $A / B$ ratios were calculated by $K M$.

The outcomes of cases including 1 and 5 min Apgar scores and long-term outcomes were separately and blindly collected by TIw, and they were reported to KM after the calculation of the $A / B$ ratio. The long-term fetal outcomes were deliberately changed into numerical values according to the dying period, the state of sickness or hospitalization (Table 2). Long-term outcomes were collected by TIw prior to the correlation study with the $A / B$ ratio.

The severity score was determined using the equation; $Y=10-7 X$, where $Y$ is $10-0$ severity and $X$ is the $A / B$ ratio. The purpose of this calculation was to easily compare the severity of fetal disorders in clinical studies.

Statistical studies were analyzed by using S. Yamazaki, The Statistical Program File of ystat2004.xls.2004, Igaku Tosho Shuppan, Tokyo.

\section{Results}

The $A / B$ ratio significantly correlated with the $1 \mathrm{~min}$ Apgar score: $R^{2}=0.85, P<0.001, Y=7.16 X-1.12$, where $Y$ was the 1 min Apgar score and $X$ was the $A / B$ ratio obtained by quantified analysis from the ACG. The 1 min Apgar scores were 6 and 3 when the $A / B$ ratios were 0.99 and 0.58 (Figure 2), where clinical 10-0 severities were 3.07 and 5.94 .

The $A / B$ ratio significantly correlated with the $5 \mathrm{~min}$ Apgar score: $\mathrm{R}^{2}=0.74, \mathrm{P}<0.001, \mathrm{Y}=6.31 \mathrm{X}+0.88$, where $Y$ was the 5 min Apgar score and $X$ was the $A / B$ ratio. The 5 min Apgar scores were 6 and 3 when the $A / B$ ratios were 0.81 and 0.34 (Figure 3 ), where $10-0$ severities were 4.33 and 7.62 .

The $A / B$ ratio significantly correlated with numerically evaluated long-term outcome: $\mathrm{R}^{2}=0.76, \quad \mathrm{P}<0.001$, $Y=-6.24 X+0.40$, where $Y$ was the numerically evaluated long-term outcome (Table 2) and $X$ was the $A / B$ ratio (Figure 4). The numerical long-term outcome was seven

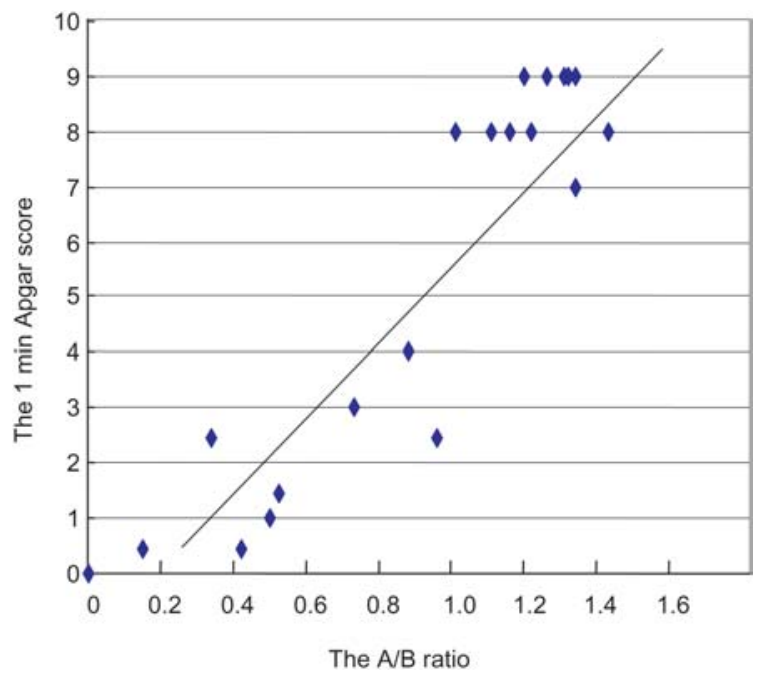

Figure 2 The A/B ratio was closely correlated to the $1 \mathrm{~min}$ Apgar score. The correlation coefficient $\left(R^{2}\right)$ was $0.85, P($ two tail) $<0.001, Y=7.16 X-1.12, n=20$, where $Y$ was the $1 \mathrm{~min}$ Apgar score and $X$ was the $A / B$ ratio.

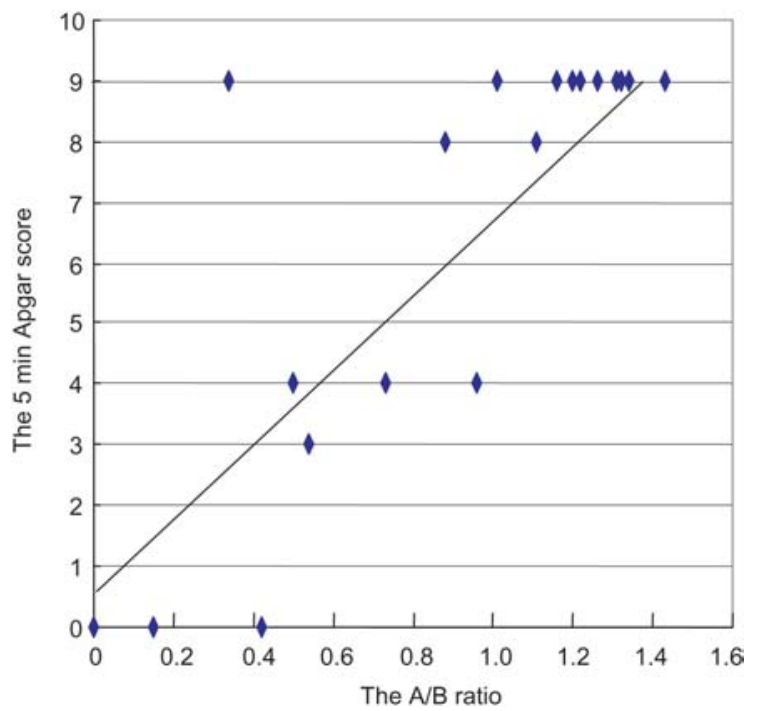

Figure 3 The $A / B$ ratio was closely correlated to the $5 \mathrm{~min}$ Apgar score. The correlation coefficient $\left(R^{2}\right)$ was 0.74 , $P$ (twotail) $<0.001, Y=6.31 X+0.88, n=20$, where $Y$ was the $5 \mathrm{~min}$ Apgar score and $X$ was the $A / B$ ratio.

(alive, mild sickness but no growth retardation) when the A/B ratio was 1.06 and the $10-0$ severity was 2.58 .

\section{Case studies}

Fetal bradycardia and normal neonate: The fetus with non-hypoxic fetal bradycardia $<60 \mathrm{bpm}$ revealed a transient FHR increase against fetal movement in the ACG where the $A / B$ ratio was as high as 1.34 and the $10-0$ severity was as low as 0.62 . An active neonate born by cesarean section with an Apgar score of 7 and whose 


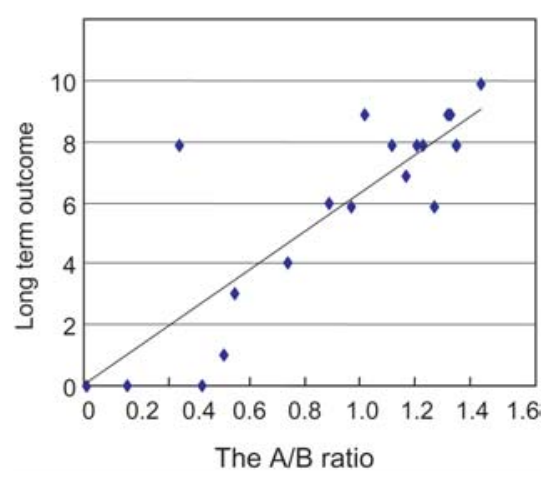

Figure 4 The A/B ratio was closely correlated to the numerically evaluated long-term outcome. The correlation coefficient $\left(R^{2}\right)$ was $0.76, P$ (two-tail) $<0.001, Y=-6.24 X+0.40, n=20$, where $\mathrm{Y}$ was the numerically evaluated long-term outcome (Table 2) and $X$ was the $A / B$ ratio.

neonatal course was favorable without a pace-maker. Fetal bradycardia was due to sick sinus syndrome [12]. The discrepancy between fetal bradycardia and a normal newborn may be understood when the importance of an accelerative FHR response to fetal movement in the ACG is considered.

FHR suggested placental insufficiency by late decelerations, and the ultrasound image revealed placental abruption, however, the $1 \mathrm{~min}$ Apgar score was 9 followed by a normal neonatal course after emergency cesarean section. The FHR which revealed late decelerations (LD) was rechecked and the $A / B$ ratio was as high as 1.32 and the $10-0$ severity was as low as 0.76 . The discrepancy between the LD pattern changes in FHR and normal newborn status may be explained by the high $\mathrm{A} / \mathrm{B}$ ratio and prompt emergency cesarean section.

Longitudinal course monitoring in high risk fetus: Severe fetal hydrops diagnosed at 26 weeks of gestation

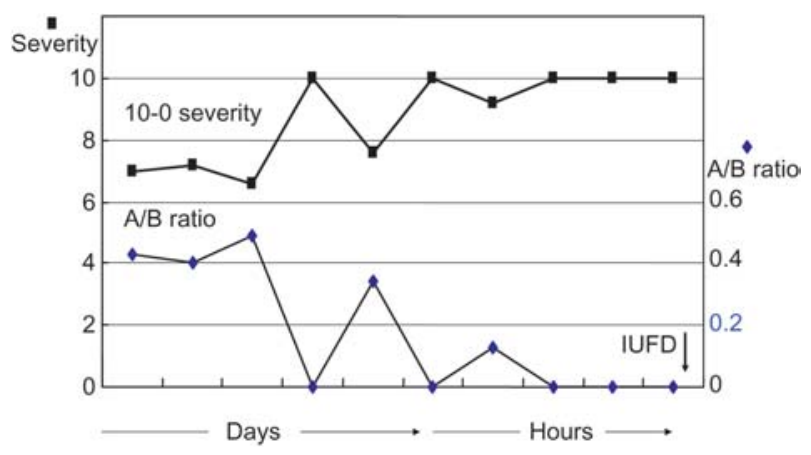

Figure 5 The longitudinal course of a fetal hydrops case demonstrates the efficacy of monitoring the changes of $A / B$ ratio and 10-0 severity in the days and hours prior to fetal demise. The gradually elevating curve is the $10-0$ severity and the scale is listed at the left side of Figure. The curve showing the downward slope is the $A / B$ ratio, the scale of which is at the right side of the Figure. was frequently monitored with ACG during the intensive treatment period, and the outcome was intrauterine demise. The A/B ratio decreased and the 10-0 severity derived from the $A / B$ ratio increased gradually demonstrating the deteriorating fetal status which was correctly evaluated by the A/B ratio during the longitudinal course (Figure 5).

\section{Discussion}

Fetal movement recognized by real-time B-mode sonography in fetal behavioral studies and biophysical profiles $[4,13,14]$ and recently 4D ultrasound promoted modern behavioral studies [6]. The ACG [7], continuously and dynamically monitors fetal movement signals and FHR with weak continuous wave Doppler ultrasound and records them on a chart [7-10]. The ACG can differentiate false positive non-reactive NST $[16,17]$ and physiological false sinusoidal FHR [5], and confirm fetal breathing and hiccupping [8, 9]. Fetal behavior was easily classified by the ACG into resting, active, intermediate and highly active states [8]. Fetal disorder severity was evaluated in this paper by the $A / B$ ratio.

At least two lines of evidence suggest that the ratio of FHR acceleration duration to fetal movement burst (A/B ratio) can predict delicate changes in severity in common fetal disorders. Firstly, ACG was able to determine the severity of fetal CNS lesions [11]. Secondly, the nonreactive NST, diagnosed by the loss of FHR acceleration against fetal movement burst in the ACG, was followed by an ominous outcome [17]. As the disappeared acceleration in the non-reactive FHR may be an extreme state, there must be various grade acceleration changes between normally frequent accelerations and its disappearance in non-reactive state.

In fact, the A/B ratio was closely and significantly correlated to the 1 and 5 min Apgar scores. An Apgar score of 3 which might represents asphyxia may be predicted by a low $A / B$ ratio such as 0.6. A 5 min Apgar score of 6 may be preceded by a low A/B ratio such as 0.8 , and a low 5 min Apgar score of 3 can be preceded by an $A$ $B$ ratio of 0.3 . In addition, numerically evaluated longterm outcome was also significantly correlated with the $A / B$ ratio calculated from the $A C G$.

The discrepancy between severe fetal bradycardia in the sick sinus syndrome and the normal status of the neonate can be explained by the fetal high A/B ratio. The discrepancy between late decelerations in placental abruption and the vigorous neonate may also be understood by the high $A / B$ ratio from the ACG recorded immediately prior to the emergency cesarean section. Therefore, the A/B ratio from the quantified ACG analysis may clarify problems in pattern analysis of the common CTG. 
In addition, the A/B ratio might be useful in longitudinal monitoring of sick fetuses during prolonged therapeutic courses. For example, the $A / B$ ratio and $10-0$ severity derived from the ratio correctly represented fetal deterioration in the course of fetal hydrops. Furthermore, longterm outcome of sick fetuses correlates with the A/B ratio. These facts suggest that the $A / B$ ratio from quantitative ACG analysis and the clinical severity derived from the $A / B$ ratio will further assist detailed evaluation in fetal disorders.

\section{References}

[1] Besinger RE, Johnson TR. Doppler recording of feta movement: clinical correlation with real-time ultrasound. Obstet Gynecol. 1989;74:277-80.

[2] de Wit AC, Nijhuis JG. Validity of the Hewlett-Packard actograph in detecting fetal movement. Ultrasound Obstet Gynecol. 2003;22:152-6.

[3] DiPietro JA, Costigan KA, Pressman EK. Fetal movement detection: comparison of the Toitu actogram with ultrasound from 20 weeks gestation. J Matern Fetal Med. 1999;8:237-42.

[4] Horimoto N, Koyanagi T, Maeda H, Satoh S, Takashima T, Nakano, $\mathrm{H}$, et al. Can brain impairment be detected by in utero behavioural patterns? Arch Dis Child. 1993;69:3-8.

[5] Ito T, Maeda K, Takahashi H, Nagata N, Nakajima K, Terakawa N. Differentiation between physiologic and pathologic sinusoidal FHR pattern by fetal actocardiogram. $J$ Perinat Med. 1994;22:39-48.

[6] Kurjak A, Stanojevic M, Andronotopo W, Salihagic-Kadic A, Carrera JM, Azumendi G. Behavioral pattern continuity from prenatal to postnatal life - a study by four-dimensional (4D) ultrasonography. J Perinat Med. 2004;32 346-53.

[7] Maeda K. Studies on new ultrasonic Doppler fetal actograph, and continuous recording of fetal movement. Nippon Sanka Fujinka Gakkai Zasshi. 1984;36:280-8.

[8] Maaeda K. Fetal monitoring and actocardiogram in the evaluation of fetal behavior. Ultrasound Rev Obstet Gynecol. 2004;4:12-25.
[9] Maeda K, Tatsumura M, Nakajima K. Objective and quantiatitive evaluation of fetal movement with ultrasonic Doppler actocardiogram. Biology of the Neonate. 1991;60(Suppl 1);41-51.

[10] Maeda K, Tatsumura M, Utsu M. Analysis of fetal movements by Doppler actocardiogram and fetal B-mode imaging. Clin Perinatol. 1999;26:829-51.

[11] Maeda K, Morokuma S, Yoshida S, Ito T, Pooh RK, Serizawa M. Fetal behavior analyzed by ultrasonic actocardiogram in fetal central nervous system lesions. J Perinat Med. 2006;34:398-403.

[12] Minagawa Y, Akaiwa A, Tsuzaki T, Tatsumura M, Ito T, Maeda K, et al. Severe fetal supraventricular bradycardia without fetal hypoxia. Obstet Gynecol. 1987;70:454-6.

[13] Morokuma S, Fukushima K, Yumoto Y, Uchimura M, Matsumoto M, Satoh S, et al. Simplified ultrasound screening for fetal brain function based on behavioral pattern. Early Hum Dev. 2007;83:177-81.

[14] Nijhuis JG, Prechtl HF, Martin GB Jr, Bots RS. Are there behavioural states in the human fetus? Early Hum Dev. 1982;6:177-95.

[15] Schwöbel E, Fallenstein F, Huch R, Huch A, Rooth G. Combined electronic fetal heart rate and fetal movement monitor - a preliminary report. J Perinat Med. 1987;15: 174-84.

[16] Stanco LM, Rabello Y, Medearis AL, Paul RH. Does Doppler-detected fetal movement decrease the incidence of non-reactive non-stress tests? Obstet Gynecol. 1993;82: 999-1003.

[17] Teshima N. Non-reactive pattern diagnosed by ultrasonic Doppler fetal actocardiogram and outcome of the fetuses with non-reactive pattern. Nippon Sanka Fujinka Gakkai Zasshi. 1993;45:423-30.

[18] Wheeler T, Robertz K, Peters J, Murrills A. Detection of fetal movement using ultrasonic Doppler ultrasound. Obstet Gynecol. 1987;70:251-4.

The authors stated that there are no conflicts of interest regarding the publication of this article.

Received October 17, 2008. Accepted November 7, 2008. Previously published online March 17, 2009. 